\title{
Ein medizinisches Potpourri
}

\section{Erhard Taverna}

erhard.taverna@saez.ch

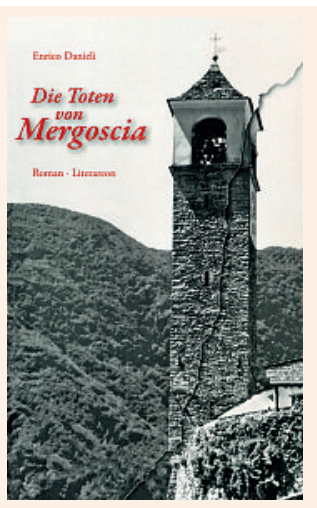

Enrico Danieli

Die Toten von Mergoscia München: Utz Verlag; 2009. 341 Seiten. Fr. 29.50 ISBN 978-3-8316-1424-0

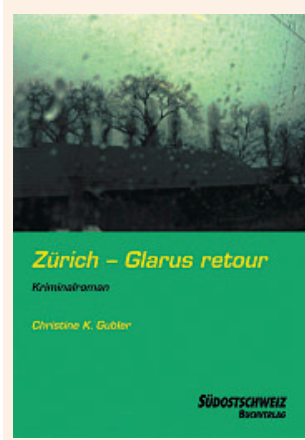

Christine K. Gubler Zürich - Glarus retour Zürich: Südostschweiz Buchverlag; 2009.

256 Seiten. Fr. 28.ISBN 978-3-905688-58-0

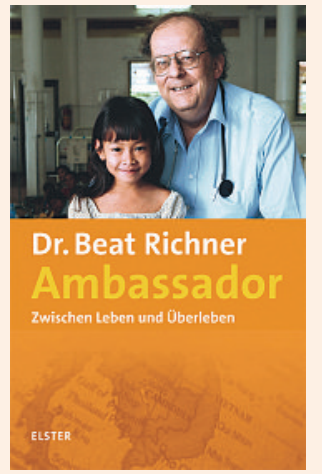

Beat Richner

Ambassador

Zürich: Elster Verlag; 2009.

192 Seiten. Fr. 36.

ISBN 978-3-907668-80-1
Fünf Autoren, eine Ärztin und vier Ärzte, liefern eine Probe ihres fachlichen und/oder schriftstellerischen Könnens. Ein ehemaliger Allgemeinpraktiker und ausgewiesener Tessinkenner erzählt die Wahngeschichte eines Besessenen, und eine praktizierende Gynäkologin führt uns im Schnellzug durch den Glarner-Filz. Ein cellospielender Kinderarzt dokumentiert sein Hilfswerk in Kambodscha, ein Hirnphysiologe schreibt ein vielbeachtetes Lehrbuch und ein spezialisierter Orthopäde den häufig nachgefragten praktischen Leitfaden zu allen komplexen Fragen der Fusschirurgie.

\section{«Die Toten von Mergoscia» von Enrico Danieli}

Leserinnen und Leser erinnern sich vielleicht noch an die Schlagzeilen zum gewaltsamen Tod der Schauspielerin Marie Trintignant 2003. Ein Fan schwört Rache, denn «sie war für mich mehr als Frau, mehr als Mensch, mehr als Schauspielerin, sie war Gott und ich ihr Anbeter». Dazu nehme man die Sommerhitze eines Filmfestivals in Locarno und den verhängnisvollen Entschluss zu einer Busfahrt in das abgelegene Bergdorf Mergoscia über dem Stausee der Verzasca. Wir begleiten den Ich-Erzähler auf seiner langen Reise, die den scheinbar Willenlosen und Getriebenen in einem Strudel aus Hass, Halluzinationen, Erinnerungen, Verdächtigungen und Nachstellungen unaufhaltsam zum tödlichen Finale mitreisst. Der Autor erschafft eine albtraumhafte Atmosphäre aus inneren Monologen und zerstörerischen Naturereignissen, aus vergeblichen Fluchtversuchen und einer zwanghaften Neugier, die jedes Entkommen aus dem Dorf vereitelt. Suchtrupps, unterstützt von Militär und Polizei, durchkämmen die Gegend nach einem vermissten Mädchen, wobei ein Täterprofil immer deutlicher hervortritt. Danieli treibt konsequent ein Verwirrspiel, legt viele Spuren aus und assoziiert kunstvoll ein Gestrüpp aus Vermutungen. Dichter als die trügerische Macchia des Tals bleiben im Roman die Indizien und Obsessionen bis zum erlösenden Ende ineinander verstrickt.

\section{«Zürich - Glarus retour» von Christine K. Gubler}

Gilles Wetter, Staatsanwalt bei der Zürcher Kantonspolizei, und froh, Ehefrau und neugeborenen Zwillingen $\mathrm{zu}$ entrinnen, unterstützt den Chef der Glarner Kantonspolizei bei der Aufklärung eines Mordes. Weil ein Zusammenhang mit einem ähnlichen Fall am Zürichberg vermutet wird, darf er täglich mit dem GlarnerSprinter in den Zigerschlitz, wo er sich, mehr als ihm lieb sein kann, im Klatsch und im Geheimnissumpf einer Kleinstadt verheddert. Christine K. Gubler, die seit über 20 Jahren als Gynäkologin im Glarnerland arbeitet, kennt die Kungeleien und Seilschaften ihres Kantons. Mit diesem Erstling ist ihr ein phantasievoll und spannend aufgebauter Krimi gelungen, dessen Haupt- und Nebenfiguren die Handlung detailreich und lebendig vorantreiben. Dass da ein Polizist im Drogenmilieu Zürichs bei seinen Ermittlungen kurz vor Ende noch seinen unbekannten Sohn entdeckt, ist vielleicht etwas zu viel des Guten. Doch Ende gut, alles gut.

\section{«Ambassador» von Beat Richner}

Seit 18 Jahren kämpft Beat Richner, inzwischen Ehrendoktor der Universitäten Lausanne und Zürich, für eine gute medizinische Versorgung der Kinder in Kambodscha. Seine Solo-Tourneen mit dem Cello sind ebenso legendär wie sein kompromissloser Kampf gegen Korruption, Politikerfilz, bürokratische Hindernisse und die Funktionärsmentalität in internationalen Gesundheitsorganisationen. Seiner Vision verdanken fünf Kantha-Bopha Spitäler ihre Existenz, tausende Kinder ihr Leben, tausende Einheimische eine Anstellung und viele weitere eine gute universitäre Ausbildung. Leidenschaft und Engagement des Arztes sind unbestritten, doch wie etwa Albert Schweitzer oder Lotti Latrous muss sich der Pionier gegen etablierte Institutionen durchsetzen, die ihre eigenen politischen und wirtschaftlichen Interessen verfolgen. WHO, DEZA, SRK und UNICEF werden unverblümt kritisiert, aber auch die Medien müssen sich Vorwürfe gefallen lassen. Spannend sind auch die Berichte aus dem Inneren des Landes, dessen Probleme wesentlich vom misslichen Umfeld mit verursacht sind. Richner scheut sich nicht, die Urheber namentlich aufzulisten.

Nur dank des im Juli 2009 neu installierten MRI wurde es möglich nachzuweisen, dass die Tuberkulose auch ausschliesslich als Hirnstammentzündung und Hirnrindenentzündung auftreten kann. Mit dem alten König Norodom Sihanouk gegen die Übermacht der amerikanischen und östlichen Militärs, gegen die Intrigen der kambodschanischen Elite, gegen miserable Privatkliniken, inkompetente NGOs und Potemkinsche Health Centers, «da muss ich mir einfach auch die Kränkungen von der Seele schreiben». Zum Glück sind da auch die bewährten Freunde in der Schweiz. Dennoch gibt es keine Beitragserhöhung für das Jahr 2010. Warum Frau Micheline Calmy-Rey nicht mehr Unterstützung zusichert, bleibt ein Rätsel. Ein grosser, einsamer Mann, der mehr als nur unseren Respekt verdient. 


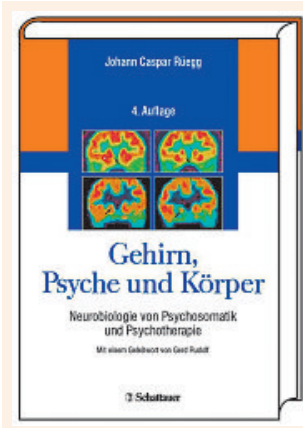

Johann Caspar Rüegg Gehirn, Psyche und Körper

Stuttgart: Schattauer; 2007. 242 Seiten. Fr. 59.90 ISBN 978-3-7945-2573-7

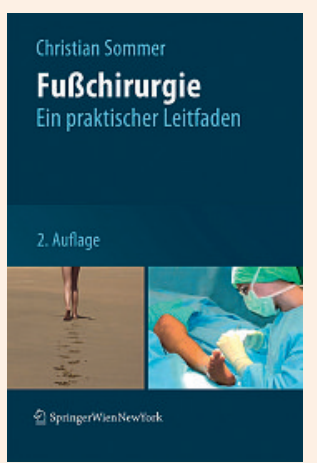

Christian Sommer (Hrsg.) Fusschirurgie.

Wien: Springer Verlag; 2010

298 Seiten. Fr. 80.90

ISBN 978-3-211-99424-5

\section{«Gehirn, Psyche und Körper» von Johann Caspar Rüiegg}

Vier Auflagen sprechen für sich. Das Lehrbuch des emeritierten Professors über die Neurobiologie von Psychosomatik und Psychotherapie ist trotz oder gerade wegen des breiten Leserkreises ein Erfolg. In sie-

\section{In sieben Kapiteln gelingt eine präzise und anregende Zusammen- fassung des aktuellen Wissens}

ben Kapiteln gelingt dem Autor eine präzise und anregende Zusammenfassung des aktuellen Wissens über neurophysiologische Grundlagen, chronische Schmerzen und Wirkung auf Herz und Kreislauf. Studien aus der Psychoendokrinologie und Psychoimmunologie über Stress, Emotionen und Hormone, Gehirn und Immunsystem, Gedächtnis und neuronale Plastizität werden kenntnisreich erläutert und auf das Wesentliche reduziert. Auch wenn die biologischen und technischen Grundlagen, unterstützt durch Abbildungen, im Mittelpunkt stehen, betont der Autor die komplexen Wechselwirkungen von Psyche und Soma, deren Verständnis die personale, interpersonelle und soziokulturelle Ebene mit einschliesst. Ausführliche Anmerkungen und Literaturangaben, sowie ein sorgfältiger Index erleichtern die Lektüre und erlauben eine gezielte Vertiefung der Inhalte.

\section{«Fusschirurgie» von Christian Sommer}

Der praktische Leitfaden des Fusschirurgen aus Luzern richtet sich, bereits in zweiter erweiterter Auflage, an alle Nichtorthopäden, an Hausärzte und Patienten. Ein ausserordentlich schön gestaltetes Buch mit einem hervorragenden Bildteil. Dem Fuss, vom Autor zu Recht als oft vernachlässigtes Körperteil bezeichnet, sind sieben ausführliche Kapitel gewidmet. Mit seinen 28 Knochen und über 50 Gelenken bezeichnet ihn Sommer als «Zehnkämpfer des Bewegungsapparates». Die Fusschirurgie hat sich mittlerweile, ähnlich der Handchirurgie, zu einem eigenständigen Fach mit einer ausgefeilten Diagnostik und über 30 Standardeingriffen entwickelt. Einführend werden die wichtigsten Grundlagen in Wort und Bild erläutert, zum Beispiel die Mechanik des Gehens, die äussere Erscheinung oder bildgebende Verfahren. Krankheitsbilder und Traumatologie sind übersichtlich nach einem klaren Schema geordnet, das konservative Methoden, Operationsrisiken und Rehabilitation mit einschliesst. Dem folgen Angaben rund um die Operation, etwa über Nahttechnik, Wundbehandlung, Verbände, Fixationen und Spezialschuhe. Beim Thema Physiotherapie finden sich auch Anweisungen zur Selbstbehandlung, und das Schlusswort gilt dem modischen (Frauen-)Schuh mit den hohen Absätzen. Das Buch ist wohltuend sachlich verfasst und zeugt von einer enormen praktischen Erfahrung. Glossar, Checkliste und Sachverzeichnis erleichtern den Gebrauch dieses Standardwerkes.

\section{Der Horror beim Ertappen eines weihnächtlichen Praxiseinbrechers}

Manfred Teichler

manfred.teichler@hin.ch
Ich hatte Notfalldienst am Samstag. In der Nacht lief so einiges. Sonntagmorgens früh ging ich schlaftrunken und ohne Brille nach unten in die winterdunkle Praxis, um das Telefon zu meinem Kollegen umzuschalten, der den Sonntagsdienst hatte. Ich machte die Tür hinter mir zu, um meine Frau nicht zu wecken. Auch habe ich kein Licht gemacht, weil ich hoffte, meine Schlaftrunkenheit nicht wegzuschocken, um mich nochmals ins warme Bett legen und Tiefschlaf nachholen zu können.

An Einbrecher dachte ich zu dieser Stunde nicht, obwohl einer vor ein paar Monaten versucht hatte, seine langen Finger um unsere Methadonvorräte zu schliessen. Zum Glück hörte meine Frau damals das Knirschen des Fensterrahmens. Die Polizei kam sehr schnell mit Hunden und verscheuchte den Schurken.
Erwischt haben sie ihn allerdings erst zwei Tage später, als er an einem anderen Ort einbrach und eine versteckte Flasche Schnaps fand, die er leertrank. Den nächsten Morgen fand man ihn, zufrieden schlafend, reif für den Knast.

Den Telefonbeantworter fertigbesprochen, drehte ich mich um: Starr vor Schreck sah ich ihn: Ein älterer ungepflegter Mann stand vor mir in der Tür und guckte mich mit grossen Augen an. Wieder ein Einbrecher! Die Angst lief mir kalt den Rücken herunter und drohte mir den Blaseninhalt nach unten zu vertreiben.

Der wilde Mann erwachte in mir, und ich reagierte sofort: Mit lautem «AAAh!» rannte ich auf ihn los, die Hände tatendurstig nach vorne gestreckt ... und rannte in den Spiegel hinein, der an der Rückseite der geschlossenen Tür befestigt ist ... 